\title{
A local fluctuation theorem
}

\author{
G. Gallavotti \\ Fisica, Università di Roma 1 \\ 13 agosto 2018
}

\begin{abstract}
A mechanism for the validity of a local version of the fluctuation theorem, uniform in the system size, is discussed for a reversible chain of weakly coupled Anosov systems.
\end{abstract}

\section{Introduction.}

In recent papers, $[\mathrm{GC}],[\mathrm{MR}]$, a general law governing the fluctuations of phase space volume contractions in a dynamical system (i.e. a smooth smoothly invertible map $S: x \rightarrow S x$ on a phase space $M)$ has been derived under various chaoticity assumptions. Its generality makes it remarkable. Similar relations had been first derived for non stationary states, [ES], and later extended to stochastic evolutions, [Ku], [LS]

The viewpoint of $[\mathrm{GC}]$ is that the reason the law holds is simply that physical systems are chaotic to the extent that one can think that they are Anosov systems, see [Ga1]: this is the chaotic hypothesis; it is a version of a general principle proposed by Ruelle, [Ru1]. ${ }^{1}$ Then, if the microscopic dynamics is time reversible, ${ }^{2}$ one can consider the fluctuations of the phase space volume contraction $\operatorname{rate}^{\mathbf{3}} \eta(x)$ averaged over a time $\vartheta$ :

$$
p=\frac{1}{\bar{\eta}_{+} \vartheta} \sum_{-\frac{1}{2} \vartheta}^{\frac{1}{2} \vartheta} \eta\left(S^{j} x\right)
$$

where $\bar{\eta}_{+}$(that we suppose $>0$, see [Ru2]) is the value of the time average of $\eta(x)$ over an infinite time $\bar{\eta}_{+}=\lim _{T \rightarrow+\infty} \frac{1}{T} \sum_{k=0}^{T-1} \eta\left(S^{j} x\right)$ : it is a quantity that is $x$-independent apart from a set of points $x$ of zero volume. We suppose, for simplicity, that the system has an attractor that is dense on phase space (this will mean, technically, that our systems are weakly interacting).

1 The hypothesis can be criticized on the basis of various results, not only on mathematical grounds but on physical grounds as well, see [Ga1], [RT].

2 i.e. there is an isometry $I$ of phase space anticommuting with time evolution and with $I^{2}=1$, see (2.1) below.

3 Equal to minus the logarithm $\eta(x)$ of the determinant of the Jacobian matrix $\partial S(x)$ of the evolution map. 
In the stationary state $\mu$, called the SRB distribution, the variable $p$ has fluctuations. Denoted $\pi_{\vartheta}(p)$ the probability distribution of $p$ in the stationary state and written it, for large $\vartheta$, as $\pi_{\vartheta}(p)=e^{\bar{\zeta}(p) \vartheta+O(1)}$, [Si], it verifies the fluctuation theorem:

$$
\frac{\bar{\zeta}(p)-\bar{\zeta}(-p)}{\bar{\eta}_{+} p}=1, \quad|p|<p^{*}
$$

for some $p^{*} \geq 1$, see $[\mathrm{GC}]$.

The phase space contraction rate is usually identified with the entropy creation rate, $[\mathrm{An}],[\mathrm{Ru} 2],[\mathrm{GC}]$. Therefore one sees that the above "law" cannot be practically verified, for physical as well as mathematical reasons: in fact the logarithm of the entropy creation rate distribution $\vartheta \bar{\zeta}(p)$ is, usually, not only proportional to $\vartheta$ but also to the spatial extension of the system, i.e. to the number of degrees of freedom; so that it is extremely unlikely that observing $p$ in a large system one can see a value $p$ which is appreciably different from 1 (note that the normalizing constant $\bar{\eta}_{+}$in (1.1) is so chosen that the average of $p$ in the stationary state is 1 ).

In macroscopic (or just "large") systems the phase space contraction rate is essentially constant (and it measures the strength of the friction) much as the density is constant in gases at equilibrium. Therefore one can hope to see entropy creation rate fluctuations only if one can define a local entropy creation rate $\eta_{V_{0}}(x)$ associated with a microscopic region $V_{0}$ of space.

In this paper we show, heuristically, why one should expect that a local entropy creation rate can be defined and verifies a local version of the fluctuation law. We defer to $§ 5$ the precise definition of local entropy creation rate as its form is not important until then.

We suppose that our system has a translation invariant spatial structure, e.g. it is a chain (or a lattice) of weakly interacting chaotic (mixing Anosov) system. Given a finite region $V_{0}$ centered at the origin and a time interval $T_{0}$, let $\eta_{+}$denote the average density of entropy creation rate, i.e. $\eta_{+}=\lim _{V_{0}, T_{0} \rightarrow \infty} \frac{1}{\left|T_{0}\right|} \frac{1}{\left|V_{0}\right|} \sum_{j=0}^{\left|T_{0}\right|-1} \eta_{V_{0}}\left(S^{j} x\right)$, then we set:

$$
p=\frac{1}{\eta_{+}|V|} \sum_{j=-\frac{1}{2}\left|T_{0}\right|}^{\frac{1}{2}\left|T_{0}\right|} \eta_{V_{0}}\left(S^{j} x\right), \quad V=V_{0} \times T_{0}
$$

where $\eta_{V_{0}}(x)$ denotes the entropy creation rate in the region $V_{0}$, a notion which we still have to define.

Calling $\pi_{V}(p)$ the probability distribution of $p$ in the stationary state $\mu$, i.e. in the SRB distribution, and assuming that the system is a weakly coupled chain of Anosov systems we show that it is $\pi_{V}(p)=e^{\zeta(p)|V|+O(|\partial V|)}$ where $|\partial V|$ denotes the size of the 
boundary of the space-time region $V$ and:

$$
\frac{\zeta(p)-\zeta(-p)}{p \eta_{+}}=1, \quad|p|<p^{*}
$$

for some $\zeta(p)$ analytic in $p$ and for some $p^{*} \geq 1$. It will also result that $\bar{\zeta}(p)=$ $r \zeta(p), \bar{\eta}_{+}=r \eta_{+}$, see (1.2), where $r$ is the ratio between the total "volume" of the system and the volume $V_{0}$, i.e. the global and local distributions are trivially related if appropriately normalized.

The interest of the above statements lies in their independence on the total size of the systems.

If $V_{0}$ is an interval of length $L=\left|V_{0}\right|$ and if $H=\left|T_{0}\right|$ then the relative size of the error and of the leading term will be, for some length $R$, of order $(L+H) R$ compared to order $L H$. Hence a relative error $O\left(H^{-1}+L^{-1}\right)$ is made by using simply $\zeta(p)$ to evaluate the logarithm of the probability of $p$ as defined by (1.3)).

This means that the fluctuation theorem leads to observable consequences if one looks at the far more probable microscopic fluctuations of the local entropy creation rate (to be yet defined: see definition in $\S 5$ below).

The key results for the present work are the papers [GC], [Ga3] and, mainly, [PS]: the latter paper provides us with a deep analysis of chains of Anosov systems and it contains, I believe, all the ingredients necessary to make the following analysis mathematically rigorous: however I do not attempt at a mathematical proof here.

\section{$\S 2$. Reversible chains of interacting Anosov systems.}

Let $\left(M^{\prime}, S^{\prime}\right)$ be a dynamical system whose phase space $M^{\prime}$ is a product of $2 N+1$ identical analytic manifolds $\bar{M}_{0}: M^{\prime}=\bar{M}_{0}^{2 N+1}$ and $S^{\prime}: M^{\prime} \rightarrow M^{\prime}$ is a small perturbation of a product map $\bar{S}_{0} \times \ldots \times \bar{S}_{0} \stackrel{\text { def }}{=} \tilde{S}_{0}$ on $M^{\prime}$. We assume that $\left(\bar{M}_{0}, \bar{S}_{0}\right)$ is a mixing Anosov system. The size $N$ (an integer) will be called the "spatial size" of the system. For $x, y, z \in \bar{M}_{0}$ let $F_{\varepsilon}(x, z, y)$ be analytic and such that $z \rightarrow F_{\varepsilon}(x, z, y)$ is a map, of $\bar{M}_{0}$ into itself, $\varepsilon$-close to the identity and $\varepsilon$-analytic for $|\varepsilon|$ small enough. We suppose that, if $\underline{x}=\left(x_{-N}, \ldots, x_{N}\right) \in M^{\prime}$ :

$$
\left(S^{\prime} \underline{x}\right)_{i}=F_{\varepsilon}\left(x_{i-1}, x_{i}, x_{i+1}\right) \circ S_{0} x_{i}
$$

where $x_{ \pm(N+1)}$ is $i d e n t i f i e d$ with $x_{\mp N}$ (i.e. we regard the chain as periodic); we call such a dynamical system a chain of interacting Anosov maps coupled by nearest neighbors. It is a special example of the class of maps considered in $[\mathrm{PS}] .{ }^{4}$

4 In the paper [PS] it is assumed that also $\bar{S}_{0}$ (hence $S_{0}$ ) is close to the identity, e.g. within $\varepsilon$ : such condition does not seem necessary for the purposes of the present paper, hence it will not be assumed. 
It is difficult, maybe even impossible, to construct a (non trivial) reversible system of the above form: we therefore (see [Ga3]) consider the system $(M, S)$ where $M=M^{\prime} \times M^{\prime}$ and define $S_{0} \stackrel{\text { def }}{=} \tilde{S}_{0} \times\left(\tilde{S}_{0}\right)^{-1}$ and $S \stackrel{\text { def }}{=} S^{\prime} \times\left(S^{\prime}\right)^{-1}$, called hereafter the free evolution and the interacting evolution, respectively. So that the system can be considered as time reversible with a time reversal map $I(\underline{x}, \underline{y})=(\underline{y}, \underline{x})$. Note that the inverse map to (2.1) does not have the same form. The map $S$ is, however, still in the class considered in [PS] because it can be written as $S(\underline{x}, \underline{y})_{i}=\left(S(\underline{x}, \underline{y})_{i 1}, S(\underline{x}, \underline{y})_{i 2}\right)$ with:

$$
\begin{aligned}
& S(\underline{x}, \underline{y})_{i 1}=F_{\varepsilon}\left(x_{i-1}, x_{i}, x_{i+1}\right) \circ S_{0} x_{i} \\
& S(\underline{x}, \underline{y})_{i 2}=G_{\varepsilon, i}(\underline{y}) \circ S_{0}^{-1} y_{i}
\end{aligned}
$$

where $G$ has "short range", i.e. $\left|G_{\varepsilon}(\underline{y})_{i}-G_{\varepsilon}\left(\underline{y}^{\prime}\right)_{i}\right|$ is of order $\varepsilon^{k}$ if $\underline{y}$ and $\underline{y}^{\prime}$ coincide on the sites $j$ with $|j-i| \leq k$. By definition the system $(M, S)$ is "reversible", i.e. :

$$
I S=S^{-1} I, \quad I^{2}=1
$$

Therefore the points of the phase space $M$ will be $(\underline{x}, \underline{y})=\left(x_{-N}, y_{-N}, \ldots, x_{N}, y_{N}\right)$ : however, to simplify notations, we shall denote them by $\underline{x}=\left(x_{-N}, \ldots, x_{N}\right)$, with $x_{j}$ denoting, of course, a pair of points in $\bar{M}_{0}$.

If $\varepsilon$ is small enough the interacting system will still be hyperbolic, i.e. for every point $\underline{x}$ it will be possible to define a stable and an unstable manifolds $W_{\underline{x}}^{s}, W_{\underline{x}}^{u}$, [PS], so that the key notion of "Markov partition", [Si] (see also [Ga2]), will make sense and it will allow us to transform, following the work [PS], the problem of studying the statistical properties of the dynamics of the system into an equivalent, but much more familiar, problem in equilibrium statistical mechanics of lattice spin systems interacting with short range forces. The reduction of the dynamical nonequilibrium problem to a short range lattice spin system equilibrium problem is the content of what follows up to $\S 5$, where the new application to a local fluctuation theorem is presented.

Let $\overline{\mathcal{P}}_{0}=\left(E_{1}^{0}, \ldots, E_{\mathcal{N}_{0}}^{0}\right)$ be a Markov partition, see [Si], for the unperturbed "single site" system $\left(\bar{M}_{0} \times \bar{M}_{0}, \bar{S}_{0} \times \bar{S}_{0}^{-1}\right)$. Then $\overline{\mathcal{P}}_{0}^{2 N+1}=\left\{E_{\alpha}\right\}, \alpha=\left(\rho_{-N}, \ldots, \rho_{N}\right)$ with $E_{\alpha}=E_{\rho_{-N}}^{0} \times E_{\rho_{-N+1}}^{0} \times \ldots \times E_{\rho_{N}}^{0}$ is a Markov partition of $\left(\bar{M}_{0}^{2(2 N+1)}, S_{0}\right)$.

The perturbation, if small enough, will deform the partition $\overline{\mathcal{P}}_{0}^{2 N+1}$ into a Markov partition $\mathcal{P}$ for $(M, S)$ changing only "slightly" the partition $\overline{\mathcal{P}}_{0}^{2 N+1}$. The work [PS] shows that the above " $\varepsilon$ small enough" mean that $\varepsilon$ has to be chosen small but that it can be chosen $N$-independent, as we shall always suppose in what follows.

Under such circumstances we can establish a correspondence between points of $M$ that have the same "symbolic history" (or "symbolic dynamics") along $\overline{\mathcal{P}}_{0}^{2 N+1}$ under $S_{0}$ and along $\mathcal{P}$ under $S$; we shall denote it by $h$; see [PS]. 


\section{$\S 3$. Operations of continuation in the symbolic dynamics.}

The Markov partition $\overline{\mathcal{P}}_{0}^{2 N+1}$ for $S_{0}$ associates with each point $\underline{x}=\left(x_{-N}, \ldots, x_{N}\right)$ a sequence $\left(\sigma_{i, j}\right), i \in[-N, N], j \in(-\infty, \infty)$ of symbols so that $\left(\sigma_{i, j}\right)_{j=-\infty}^{\infty}$ is the free symbolic dynamics of the point $x_{i}$. We call the first label $i$ of $\sigma_{i, j}$ a "space-label" and the second a "time-label". Not all sequences can arise as histories of points: however (by the definition of $h$, see $\S 2$ ) precisely the same sequences arise as histories of points along $\mathcal{P}_{0}$ under the free evolution $S_{0}$ or along $\mathcal{P}$ under the interacting evolution $S$.

The map $h$ is Hölder continuous and "short ranged":

$$
\left|h(\underline{x})_{i}-h\left(\underline{x}^{\prime}\right)_{i}\right| \leq C \sum_{j} \varepsilon^{|i-j| \gamma^{\prime}}\left|x_{j}-x_{j}^{\prime}\right|^{\gamma}
$$

for some $\gamma, \gamma^{\prime}, C>0$, [PS], if $|x-y|$ denotes the distance in $\bar{M}_{0} \times \bar{M}_{0}$ (i.e. in the single site phase space).

Furthermore the code $\underline{x} \longleftrightarrow \underline{\sigma}$ associating with $\underline{x}$ its "history" or "symbolic dynamics" $\underline{\sigma}(\underline{x})$ along the partition $\mathcal{P}$ under the map $S$ is such that, fixed $j$ :

$$
\underline{\sigma}(\underline{x})_{i}=\underline{\sigma}\left(\underline{x}^{\prime}\right)_{i} \text { for }|i-j| \leq \ell \quad \Rightarrow \quad\left|x_{j}-x_{j}^{\prime}\right| \leq C \varepsilon^{\gamma \ell}
$$

The inverse code associating with a history $\underline{\sigma}$ a point with such history will be denoted $\underline{x}(\underline{\sigma})$.

If $\underline{x}=\left(x_{-N}, \ldots, x_{N}\right)$ is coded into $\underline{\sigma}(\underline{x})=\left(\underline{\sigma}_{-N}, \ldots, \underline{\sigma}_{N}\right)=\left(\sigma_{i, j}\right)$, with $i=$ $-N, \ldots, N$, and $j \in(-\infty,+\infty)$, the short range property holds also in the time direction. This means that, fixed $i_{0}$ :

$$
\sigma_{i, j}=\sigma_{i, j}^{\prime} \text { for }\left|i-i_{0}\right|<k,|j|<p \quad \Rightarrow \quad\left|\underline{x}(\underline{\sigma})_{i_{0}}-\underline{x}\left(\underline{\sigma}^{\prime}\right)_{i_{0}}\right| \leq C \varepsilon^{\gamma k} e^{-\kappa p}
$$

for some $\kappa, \gamma, C>0$, [PS] lemma 1 . The constants $\kappa, \gamma, C, C^{\prime}, B, B^{\prime}>0$ above and below should not be thought to be the same even when denoted by the same symbol: however they could be a posteriori fixed so that to equal symbols correspond equal values.

By construction the codes $\underline{x} \longleftrightarrow \underline{\sigma}(\underline{x})$ commute with time evolution.

The sequences $\left(\sigma_{i, j}\right)$ which arise as symbolic dynamics along $\overline{\mathcal{P}}_{0}$ under the free single site evolution of a point $x_{i}$ are subject to constraints, that we call "vertical", imposing that $T_{\sigma_{i, j}, \sigma_{i, j+1}}^{0} \equiv 1$ for all $j$, if $T_{\sigma, \sigma^{\prime}}^{0}$ denotes the "compatibility matrix" of the "free single site evolution" (i.e. $T_{\sigma, \sigma^{\prime}}^{0}=1$ if the $\bar{S}_{0} \times \bar{S}_{0}^{-1}$ image of $E_{\sigma}$ intersects the interior of $E_{\sigma^{\prime}}$ and $T_{\sigma, \sigma^{\prime}}^{0}=0$ otherwise). We call the latter condition a "compatibility condition" for the spins in the $i$-th column. 
The mixing property of the free evolution immediately implies that a large enough power of the compatibility matrix $T^{0}$ has all entries positive. This means that for each symbol $\sigma$ we can find semiinfinite sequences:

$$
\begin{aligned}
& \sigma_{B}(\sigma)=\left(\ldots, \sigma_{-1}, \sigma_{0} \equiv \sigma\right), \quad T_{\sigma_{i-1}, \sigma_{i}}^{0}=1, \quad \text { for all } i \leq 0 \\
& \sigma_{T}(\sigma)=\left(\sigma \equiv \sigma_{0}, \sigma_{1}, \ldots\right), \quad T_{\sigma_{i}, \sigma_{i+1}}^{0}=1, \quad \text { for all } i \geq 0
\end{aligned}
$$

and defines two functions $\sigma_{B}, \sigma_{T}$, called "compatible extensions", defined on the set $\left\{1, \ldots, \mathcal{N}_{0}\right\}$ of labels of the single site Markov partition $\overline{\mathcal{P}}_{0}$, with values in the compatible semiinfinite sequences.

In fact there are (uncountably) many ways of performing such compatible extensions "from the bottom" and "from the top" of the symbol $\sigma$ into semiinfinite compatible sequences of symbols. We imagine to select one pair $\sigma_{B}, \sigma_{T}$ arbitrarily, once and for all, and call such a selection a "choice of boundary conditions" or "of extensions", on symbolic dynamics, for reasons that should become clear shortly.

We shall therefore be able to "extend in a standard way" any finite compatible block ${ }^{5}$ $Q$ of spins:

$$
\underline{\sigma}_{Q}=\left(\sigma_{i, j}\right)_{i \in L, j \in K}, \quad L=(a-\ell, a+\ell), K=(b-m, b+m)
$$

by setting $\sigma_{i, j}=\sigma_{B}\left(\sigma_{i, b-n}\right)_{b-n-j}$ for $j<b-n$ and $\sigma_{i, j}=\sigma_{T}\left(\sigma_{i, b+n}\right)_{j-b-n}$ for $j>b+n$. Here $a, b, \ell, m$ are integers.

In the free evolution there are no "horizontal" compatibility constraints; hence it is always possible to extend the finite block $\underline{\sigma}_{Q}=\left(\sigma_{i, j}\right)_{i \in L, j \in K}$ to a "full spin configuration" sequence $\left(\sigma_{i, j}\right)_{i \in[-N, N], j \in(-\infty, \infty)}$, obtained by continuing the columns in the just described standard way, using the boundary extensions $\sigma_{B}, \sigma_{T}$, above the top and below the bottom, into a biinfinite sequence and also by extending the spin configuration to the right and to the left to a sequence with spatial labels running over the full spatial range $[-N, N]$. One simply defines $\sigma_{i, j}$ for $i \notin L$ as any (but prefixed once and for all) compatible biinfinite sequence of symbols (the same for each column).

The allowed symbolic dynamics sequences for the free dynamics (on $\mathcal{P}_{0}$ ) and for the interacting dynamics (on $\mathcal{P}$ ) coincide because the free and the interacting dynamics are conjugated by the map $h$, [PS]. Therefore the above operations make sense also if the sequences are regarded as symbolic sequences of the interacting dynamics, as we shall do from now on.

To conclude: given a "block" $\underline{\sigma}_{Q}$ of symbols, with space-time labels $(i, j) \in Q=L \times K$, we can associate with it a point $\underline{x} \in M$ whose symbolic dynamics is the above described

5 A block $\left(\sigma_{i, j}\right),(i, j) \in Q$, is naturally said to be "compatible" if $T_{\sigma_{i, j}, \sigma_{i, j+1}}^{0}=1$ for all $(i, j) \in Q$ such that $(i, j+1)$ is also in $Q$. 
standard extension of $\underline{\sigma}_{Q}$. The latter depends only on the values of $\sigma_{i, j}$ for $j$ at the top or at the bottom of $Q$ and, of course, on the boundary conditions $\sigma_{B}, \sigma_{T}$ chosen to begin with.

\section{$\S 4$. Expansion and contraction rates.}

Consider the rates of variation of the phase space volume, $\lambda_{0}(\underline{x})$, or, respectively, of the surface elements of the stable and unstable manifolds $\lambda_{s}(\underline{x})$ and $\lambda_{u}(\underline{x})$ at the point $\underline{x}$ : they are the logarithms of the Jacobian determinants $\partial S(\underline{x}), \partial_{(\alpha)} S(\underline{x}), \alpha=s, u$, where $\partial_{(\alpha)}$ denotes the Jacobian of $S$ as a map of $W_{\underline{x}}^{\alpha}$ to $W_{S \underline{x}}^{\alpha}$ where $\alpha=u, s$ distinguishes the unstable manifold $W_{\underline{x}}^{u}$ of $\underline{x}$ or the stable manifold $\bar{W}_{\underline{x}}^{\sigma}$ of $\underline{x}$ :

$$
\lambda_{\alpha}(\underline{x})=-\log \left|\operatorname{det} \partial_{(\alpha)} S(\underline{x})\right|, \quad \alpha=0, u, s
$$

where $\partial_{(0)} S(\underline{x}) \stackrel{\text { def }}{=} \partial S(\underline{x})$.

A hard technical problem is to represent $\lambda_{\alpha}(\underline{x})$ in terms of the "symbolic history" of $\underline{x}$ along $\mathcal{P}$, i.e. in terms of compatible sequences $\underline{\sigma}=\left(\sigma_{i, j}\right)$ with $i \in(-N, N), j \in$ $(-\infty, \infty)$. The rates $\lambda_{\alpha}(\underline{x})$ can be expressed as:

$$
\lambda_{\alpha}(\underline{x})=-\log \left|\operatorname{det} \frac{\partial S}{\partial \underline{x}}\right|_{W^{\alpha}(\underline{x})}=\sum_{L \subset[-N, N]} \tilde{\delta}_{L}^{(\alpha)}\left(\underline{x}_{L}\right)
$$

where $L$ is an interval in $[-N, N]$ (with $\pm(N+1)$ identified with $\mp N)$, [PS].

For $\alpha=0$ this can be done by noting that the matrix $J=\frac{\partial S}{\partial x}$ has an almost diagonal structure: $J(\underline{x})=J_{0}(\underline{x})(1+\Delta(\underline{x}))$ where $J_{0}(\underline{x})$ is the Jacobian matrix of the free motion $J_{0}(\underline{x})=\bar{J}_{0}\left(x_{-N}\right) \times \bar{J}_{0}\left(x_{-N+1}\right) \times \ldots \times \bar{J}_{0}\left(x_{N}\right)$ if $\underline{x}=\left(x_{-N}, \ldots, x_{N}\right)$ and if $D=\left(\prod_{j=-N}^{N} \operatorname{det} \bar{J}_{0}\left(x_{j}\right)\right)$ :

$$
\operatorname{det} J=D \cdot e^{\operatorname{Tr} \log (1+\Delta(\underline{x}))}=D \cdot e^{\sum_{k=1}^{\infty} \frac{(-1)^{k-1}}{k} \operatorname{Tr} \Delta(\underline{x})^{k}}
$$

which leads to (4.2) if one uses that the matrix elements $\Delta_{p, q}=J_{0}^{-1}(\underline{x}) \partial_{x_{p}} \partial_{x_{q}} J(\underline{x})$ are essentially local, i.e. bounded by $B(C \varepsilon)^{|p-q| \gamma}$ for some $\gamma, C, B>0$ (see (2.1),(2.2), $(3.3))$.

For $\alpha=u, s(4.2)$ can be derived in a similar way using also that:

(1) the stable and unstable manifolds of $\underline{x}$ consist of points $\underline{y}$ which have eventually, respectively towards the future or towards the past, the same history of $\underline{x}$,

(2) they are described in a local system of coordinates around $\underline{x}=\left(\ldots, x_{-1}, x_{0}, x_{1}, \ldots\right)$ by smooth "short range" functions. Suppose, in fact, that on each factor $M_{0}$ we introduce a local system of coordinates $(\alpha, \beta)$ around the point $x_{i} \in M_{0}$, such that the 
unperturbed stable and unstable manifolds are described locally by graphs $\left(\alpha, f_{s}(\alpha)\right)$ or $\left(f_{u}(\beta), \beta\right)$.

The unperturbed stable and unstable manifolds will be smooth graphs $\left(\alpha_{i}, f_{s}\left(\alpha_{i}\right)\right)$ or $\left(f_{u}\left(\beta_{i}\right), \beta_{i}\right)$ with $\alpha_{i}$ varying close to $\bar{\alpha}_{i}$ and $\beta_{i}$ close to $\bar{\beta}_{i}$, with $\left(\bar{\alpha}_{i}, \bar{\beta}_{i}\right)$ being the coordinates of $x_{i}$.

Fixed a point $\underline{x}=\left(x_{-N}, \ldots, x_{N}\right)$ with coordinates $\left(\bar{\alpha}_{i}, \bar{\beta}_{i}\right)_{i=-N, \ldots, N}$ the perturbed manifolds of the point $\underline{x}$ will be described by smooth (at least $C^{2}$ and in fact of any prefixed smoothness if $\varepsilon$ is sufficiently small) functions $W^{s}(\underline{\alpha}), W^{u}(\underline{\beta})$ of $\underline{\alpha}=\left(\alpha_{i}\right)_{i=-N, N}$ or of $\underline{\beta}=\left(\beta_{i}\right)_{i=-N, N}$ which are "local"; i.e. if $\underline{\alpha}$ and $\underline{\alpha}^{\prime}$ agree on the sites $i-\ell, i+\ell$ or if $\underline{\beta}$ and $\underline{\beta}^{\prime}$ agree on the sites $i-\ell, i+\ell$ then:

$$
\begin{array}{ll}
\left\|W^{u}(\underline{\beta})_{i}-f_{u}\left(\beta_{i}\right)\right\|_{C^{2}}<C \varepsilon, & \left\|W^{u}(\underline{\beta})_{i}-W^{u}\left(\underline{\beta}^{\prime}\right)_{i}\right\|_{C^{2}}<C \varepsilon^{\ell} \\
\left\|W^{s}(\underline{\alpha})_{i}-f_{s}\left(\alpha_{i}\right)\right\|_{C^{2}}<C \varepsilon, & \left\|W^{s}(\underline{\alpha})_{i}-W^{s}\left(\underline{\alpha}^{\prime}\right)_{i}\right\|_{C^{2}}<C \varepsilon^{\ell}
\end{array}
$$

for some $C>0$, see [PS] lemmata 1,2. Here the norms in the first column are the norms in $C^{2}$ as functions of the arguments $\underline{\beta}$ or respectively $\underline{\alpha}$, while the norms in the second column are $C^{2}$ norms evaluated (of course) after identifying the arguments of $\underline{\beta}$ (or $\underline{\alpha}$ ) and $\underline{\beta}^{\prime}$ (or $\underline{\alpha}^{\prime}$ ) with labels $j$ such that $|i-j| \leq \ell$.

(3) If we consider the dependence of the planes tangent to the stable and unstable manifolds $W_{\underline{x}}^{s}, W_{\underline{x}}^{u}$ at $\underline{x}$ we find that they are Hölder continuous as functions of $\underline{x}$ :

$$
\left|\left(d W_{\underline{x}}^{\alpha}\right)_{i}-\left(d W_{\underline{y}}^{\alpha}\right)_{i}\right|<C \sum_{j} \varepsilon^{|i-j| \kappa}\left|x_{j}-y_{j}\right|^{\gamma}, \quad \alpha=u, s
$$

where $\left(d W_{\underline{x}}^{\alpha}\right)_{i}$ denoted the components relative to the $i$-th coordinate of $\underline{x}$ of the tangent plane to $W_{\underline{x}}^{\alpha}$ and $C, \kappa, \gamma>0$.

The above properties and the Hölder continuity (3.1), (3.2), (3.3) imply that the "horizontal potentials" $\tilde{\delta}_{L}^{(\alpha)}\left(\underline{x}_{L}\right)$ in (4.2) are "short ranged":

$$
\left|\tilde{\delta}_{L}^{(\alpha)}\left(\underline{x}_{L}\right)\right| \leq B(C \varepsilon)^{(|L|-1) \gamma}, \quad \alpha=u, s
$$

for some $B, C, \gamma>0$; we denote $|L|$ the number of points in the set $L$.

We shall use the symbolic representation of $\underline{x} \in M$ to express the rates $\lambda^{(\alpha)}(\underline{x})$. For this purpose let $\underline{x}=\left(x_{i}\right)_{i=-N, N}$ and suppose that such $\underline{x}$ corresponds to the symbolic dynamics sequence $\underline{\sigma}=\left(\underline{\sigma}_{j}\right)_{j=-\infty}^{\infty}$ where $\underline{\sigma}_{j}=\left(\sigma_{-N, j}, \ldots, \sigma_{N, j}\right)$. We denote $\underline{\sigma}_{L}$ the sequence $\underline{\sigma}_{L}=\left(\sigma_{i, j}\right)_{i \in L, j=-\infty, \infty}$.

Then $\underline{\sigma}_{L}$ does not determine $\underline{x}_{L}$ (unless there is no interaction, i.e. $\varepsilon=0$ ): however the short range property, (3.3), of the symbolic codes and of the map $h$ conjugating the free evolution and the interacting evolution shows that, if $L^{\prime}$ is a larger interval 
containing $L$ and centered around $L$, then the sequence $\underline{\sigma}_{L^{\prime}}$ determines each point of $\underline{x}_{L}$ within an approximation $\leq(C \varepsilon)^{\left(\left|L^{\prime}\right|-|L|\right) \gamma}$. Hence we can define $\widehat{\delta}_{L}^{(\alpha)}\left(\underline{\sigma}_{L}\right)$ so that:

$$
\begin{aligned}
\tilde{\delta}_{L}^{(\alpha)}\left(\underline{x}_{L}\right) & =\sum_{L^{\prime} \supset L} \widehat{\delta}_{L^{\prime}}^{(\alpha)}\left(\underline{\sigma}_{L^{\prime}}\right), \quad\left|\widehat{\delta}_{L}^{(\alpha)}\left(\underline{\sigma}_{L}\right)\right|<B^{\prime}\left(C^{\prime} \varepsilon^{\gamma}\right)^{|L|-1} \\
\lambda_{\alpha}(\underline{x}) & =\sum_{L} 2^{|L|} \widehat{\delta}_{L}^{(\alpha)}\left(\underline{\sigma}_{L}\right)
\end{aligned}
$$

for some $B^{\prime}, C^{\prime}, \gamma$. This leads to expressing $\lambda_{\alpha}(\underline{x})$ in terms of the symbolic dynamics of $\underline{x}$ and of the "space-localized" potentials $\widehat{\delta}_{L}^{(\alpha)}\left(\underline{\sigma}_{L}\right)$.

Let $Q_{n}=L \times K$ where $K=[-n, n]$ is a "time-interval" and set

$$
\mathcal{L}_{Q_{n}}^{\alpha}\left(\underline{\sigma}_{Q_{n}}\right) \stackrel{\text { def }}{=} \widehat{\delta}_{L}^{(\alpha)}\left(\left[\underline{\sigma}_{Q_{n}}\right]\right)-\widehat{\delta}_{L}^{(\alpha)}\left(\left[\underline{\sigma}_{Q_{n-1}}\right]\right)
$$

if $n \geq 1$ and $\left[\underline{\sigma}_{Q_{n}}\right]$ denotes a standard extension (in the sense of $\S 3$ ) of $\underline{\sigma}_{Q_{n}}$; or just set $\mathcal{L}_{Q_{0}}^{\alpha} \stackrel{\text { def }}{=} \widehat{\delta}_{L}^{(\alpha)}\left(\left[\underline{\sigma}_{Q_{0}}\right]\right)$ for $n=0$. We define $\mathcal{L}_{Q}^{\alpha}\left(\underline{\sigma}_{Q}\right)$ for $Q=L \times K$ and $K$ not centered (i.e. $K=(a-n, a+n), a \neq 0)$ so that it is translation invariant with respect to space time translations (of course the horizontal translation invariance is already implied by the above definitions and the corresponding translation invariance of $\widetilde{\delta}_{L}^{(\alpha)}$ ).

The remarkable property, consequence of the Hölder continuity of the functions in (4.1) and of the (3.3),(4.7), see [PS], is that for some $\gamma, \kappa, B, C>0$ :

$$
\left|\mathcal{L}_{Q}^{\alpha}\left(\underline{\sigma}_{Q}\right)\right| \leq B\left(C \varepsilon^{\gamma}\right)^{i} e^{-\kappa j}
$$

if $i, j$ are the horizontal and vertical dimensions of $Q$.

In this way we define a "space-time local potential" $\mathcal{L}_{Q}^{(\alpha)}$ which is, by construction, translation invariant and such that, if $\Lambda$ denotes the box $\Lambda=[-N, N] \times[-M, M]$ the following representations for the rates in (4.1) hold:

$$
-\log \left|\operatorname{det} \partial_{(\alpha)} S^{2 M+1}\left(S^{-M} \underline{x}\right)\right|=\sum_{Q \subset \Lambda} \mathcal{L}_{Q}^{\alpha}\left(\underline{\sigma}_{Q}\right)+O(|\partial \Lambda|)
$$

where $O(|\partial \Lambda|)$ is a "boundary correction" due to the fact that in (4.10) one should really extend the sum over the $Q$ 's centered at height $\leq M$ and contained in the infinite strip $[-N, N] \times[-\infty, \infty]$ rather than restricting $Q$ to the region $\Lambda$. Hence the remainder in (4.10) can, in principle, be explicitly written, in terms of the potentials $\mathcal{L}_{Q}^{(\alpha)}$, in the boundary term form usual in Statistical Mechanics of the 2-dimensional short range Ising model and it can be estimated to be of $O(|\partial \Lambda|)$ by using (4.9). 


\section{$\S 5$. Symmetries. Local entropy creation. SRB states and fluctuations.}

Besides the obvious translation invariance symmetry the dynamical system has a time reversal symmetry; this is the diffeomorphism $I$, see (2.3), which anticommutes with $S$ and $S_{0}$ :

$$
I S=S^{-1} I, \quad I S_{0}=S_{0} I^{-1}, \quad I^{2}=1
$$

We can suppose that the Markov partition is time reversible, i.e. to each element $E_{\underline{\sigma}}$ of the partition $\mathcal{P}$ one can associate an element $E_{\underline{\sigma}^{\prime}}=I E_{\underline{\sigma}}$ which is also an element of the partition. Here we simply use the invariance of the Markov partition property under maps that either commute or anticommute with the evolution $S$ : hence it is not restrictive, see [Ga2],[Ga3], to suppose that for each $\underline{\sigma}$ one can define a $\underline{\sigma}^{\prime}$ so that $E_{\underline{\sigma}^{\prime}}=I E_{\underline{\sigma}}$. We shall denote such $\underline{\sigma}^{\prime}$ as $I \underline{\sigma}$ or also $-\underline{\sigma}$. For $\varepsilon=0$, i.e. for vanishing perturbation, the map $I$ will act independently on each column of spins of $\underline{\sigma}$. This property remains valid for small perturbations; hence:

$$
I \underline{\sigma}=\left\{\sigma_{i, j}^{\prime}\right\}=\left\{-\sigma_{i,-j}\right\} \stackrel{\text { def }}{=}-\underline{\sigma}^{I}
$$

i.e. time reversal simply reflects the spin configuration corresponding to a phase space point and changes "sign" of each spin.

The functions $\lambda_{\alpha}(\underline{x})$ and their "potentials" $\mathcal{L}_{Q}^{\alpha}\left(\underline{\sigma}_{Q}\right)$ verify, as a consequence, if $Q=$ $[-\ell, \ell] \times[-k, k]$ is a centered rectangle:

$$
\lambda_{\alpha}(I \underline{x})=-\lambda_{\alpha^{\prime}}(\underline{x}), \quad \mathcal{L}_{Q}^{\alpha}\left(\underline{\sigma}_{Q}\right)=-\mathcal{L}_{Q}^{\alpha^{\prime}}\left(-\underline{\sigma}_{Q}^{I}\right)
$$

where $\alpha^{\prime}=s$ if $\alpha=u$ and $\alpha^{\prime}=u$ if $\alpha=s, \alpha^{\prime}=0$ if $\alpha=0$. The above symmetries will be translated into remarkable properties of the SRB distribution.

Definition: Fixed a point $\underline{x}=\left(\ldots, x_{\ell-1}, x_{\ell}, x_{\ell+1}, \ldots\right)$ consider the map (2.1) as a map of $\underline{x}_{V_{0}} \stackrel{\text { def }}{=}\left(x_{j}\right)_{j \in V_{0}}=\left(x_{-\ell}, \ldots, x_{\ell}\right)$ into:

$$
x_{V_{0}}^{\prime}=S\left(\ldots, x_{-\ell-1}, \underline{x}_{V_{0}}, x_{\ell+1}, \ldots\right)_{V_{0}}
$$

defined by (2.1) for $i \in[-\ell, \ell]$. We call "local entropy production rate" associated with the "space like box" $V_{0}=[-\ell, \ell]$ at the phase space point $\underline{x}=\left(\ldots, x_{\ell-1}, x_{\ell}, x_{\ell+1}, \ldots\right)$ the quantity $\eta_{V_{0}}^{0}(\underline{x})$ equal to minus the logarithm of the determinant of the $2(2 \ell+1) \times$ $2(2 \ell+1)$ Jacobian matrix of the map, (5.4).

Likewise we can consider the corresponding Jacobian determinants of the restriction of the map $S$ to the stable and unstable manifolds of $\underline{x}$. Such determinants will depend not only from $x_{i}, i \in V_{0}$, and on the nearest neighbors variables $x_{ \pm \ell}$ but also on the 
other ones $x_{k}$ with $|k|>\ell+1$ : however their dependence from the variables with labels $|k|>\ell$ is exponentially damped as $\varepsilon^{(|k|-\ell) \gamma}$, by (4.9).

If we look at the average phase space variation rates $\eta_{V_{0}}^{0}, \eta_{V_{0}}^{s}, \eta_{V_{0}}^{u}$ between the time $-\vartheta$ and $\vartheta$ we can find, via a power expansion like the one in (4.3) along the lines leading from (4.3) to (4.10), a mathematical expression as:

$$
\eta_{V_{0}}^{\alpha}(\underline{x}) \simeq \sum_{Q}{ }^{*} \mathcal{L}_{Q}^{\alpha}\left(\underline{\sigma}_{Q}\right)
$$

where the $\sum_{Q}^{*} Q$ runs over rectangles $Q$ centered at 0 -time $Q=[a-\ell, a+\ell] \times[-k, k]$ with $[a-\ell, a+\ell] \subseteq V_{0}$. This could be taken as an alternative definition of $\eta_{V_{0}}^{\alpha}$, as it is a rather natural expression. For our purposes, if $V=V_{0} \times[-\vartheta, \vartheta]$, one needs to note that (5.5) holds at least in the sense that:

$$
\frac{1}{V_{0} \cdot(2 \vartheta+1)} \sum_{j=-\vartheta}^{\vartheta} \eta_{V_{0}}^{(\alpha)}\left(S^{j} \underline{x}\right)=\frac{1}{V_{0} \cdot(2 \vartheta+1)} \sum_{Q \subset V} \mathcal{L}_{Q}^{\alpha}(\underline{\sigma} Q)+\frac{O(|\partial V|)}{|V|}
$$

i.e. expression (5.5) can be used to compute the average local entropy creation rate in the space-time region $V$ up to boundary corrections $O(|\partial V|$ ) (that can be neglected for the purposes of the following discussion).

We now study the SRB distribution $\mu$ : denoting by $\langle F\rangle_{+}$the average value with respect to $\mu$ of the observable $F$ we can say, see [Si], $[\mathrm{PS}]$, that if $\Lambda=[-N, N] \times[-T, T]$ :

$$
\langle F\rangle_{+}=\lim _{T \rightarrow \infty} \frac{\sum_{\underline{\sigma}} F(\underline{\sigma}) e^{\sum_{Q \subset \Lambda} \mathcal{L}_{Q}^{u}\left(\underline{\sigma}_{Q}\right)}}{\sum_{\underline{\sigma}} e^{\sum_{Q \subset \Lambda} \mathcal{L}_{Q}^{u}\left(\underline{\sigma}_{Q}\right)}}
$$

We want to study the properties of the fluctuations of:

$$
p=\frac{1}{V \eta_{+}} \sum_{Q \subset V} \mathcal{L}_{Q}^{u}\left(\underline{\sigma}_{Q}\right), \quad \text { if } \quad \eta_{+}=\lim _{V \rightarrow \infty} \frac{1}{V} \sum_{Q \subset V}\left\langle\mathcal{L}_{Q}^{u}\right\rangle_{+}
$$

for which we expect a distribution of the form $\pi_{V}(p)=$ const $e^{V \zeta(p)+O(\partial V)}$. The SRB distribution gives to the event that $p$ is in the interval $d p$ the probability $\pi_{V}(p) d p$ with:

$$
\pi_{V}(p)=\text { const } \sum_{\text {at } \text { fixed }_{p}} e^{\sum_{Q \subset \Lambda} \mathcal{L}_{Q}^{u}\left(\underline{\sigma}_{Q}\right)}
$$

and (defining implicitly $\left.U^{u}\right)$ :

$$
\begin{aligned}
& \sum_{Q \subset \Lambda} \mathcal{L}_{Q}^{u}\left(\underline{\sigma}_{Q}\right)=\sum_{Q \subset V} \mathcal{L}_{Q}^{u}\left(\underline{\sigma}_{Q}\right)+\sum_{Q \subset \Lambda / V} \mathcal{L}_{Q}^{u}\left(\underline{\sigma}_{Q}\right)+O\left(|\partial V| \kappa^{-1}\right) \stackrel{\text { def }}{=} \\
& \stackrel{\text { def }}{=} U_{V}^{u}\left(\underline{\sigma}_{V}\right)+U_{\Lambda / V}^{u}\left(\underline{\sigma}_{\Lambda / V}\right)+O\left(|\partial V| \kappa^{-1}\right)
\end{aligned}
$$


with $\kappa>0$, having used the "short range" properties (4.9) of the potential.

In the sums in (5.7) we would like to sum over $\underline{\sigma}_{V}$ and over $\underline{\sigma}_{\Lambda / V}$ as if such spins were independent labels. This is not possible because of the vertical compatibility constraints. However the mixing property supposed on the free evolution implies that the compatibility matrix $T^{0}$ raised to a large power $R$ has positive entries. Hence if we leave a gap of width $R$ above and below $V$ we can regard as independent labels the labels $\sigma_{i, j}$ with $i$ in the space part $V_{0}$ of the region $V=V_{0} \times[-\vartheta, \vartheta]$ and with $|j|>\vartheta+R$, by a distance $\geq R$ above or below the region $V$. Denoted $V+R \stackrel{\text { def }}{=} V_{0} \times[-\vartheta-R, \vartheta+R]$ remark that:

$$
\sum_{Q \subset \Lambda} \mathcal{L}_{Q}^{u}\left(\underline{\sigma}_{Q}\right)=U_{V}^{u}\left(\underline{\sigma}_{V}\right)+U_{\Lambda /(V+R)}^{u}\left(\underline{\sigma}_{\Lambda /(V+R)}\right)+O\left(|\partial V|\left(R+\kappa^{-1}\right)\right)
$$

Hence, proceeding as in [GC1], we change the sum over (the dummy label) $\underline{\sigma}$ in the denominator to a sum over $-\underline{\sigma}^{I}$ and using $\mathcal{L}_{Q^{I}}^{u}\left(-\underline{\sigma}_{Q}^{I}\right)=-\mathcal{L}_{Q}^{s}\left(\underline{\sigma}_{Q}\right)$ :

$$
\frac{\pi_{V}(p)}{\pi_{V}(-p)}=\frac{\sum_{\text {at fixed } p} e^{\sum_{Q \subset V} \mathcal{L}_{Q}^{u}\left(\underline{\sigma}_{Q}\right)} e^{U_{\Lambda /(V+R)}^{u}\left(\underline{\sigma}_{\Lambda /(V+R)}\right)}}{\sum_{\text {at fixed } p} e^{\sum_{Q \subset V}-\mathcal{L}_{Q}^{s}\left(\underline{\sigma}_{Q}\right)} e^{U_{\Lambda /(V+R)}^{u}\left(\left(-\underline{\sigma}^{I}\right)_{\Lambda /(V+R)}\right)}} e^{O(|\partial V|)}
$$

with summation being over spin configurations in the "whole space-time" $\Lambda$, subject to the specified constraint of having the same value for $p$, i.e. same average local entropy creation rate in the space-time region $V$. The latter expression becomes, since labels $\underline{\sigma},-\underline{\sigma}^{I}$ (resp. in numerator and denominator of (5.12)) are independent dummy labels:

$$
\frac{\sum_{\text {at fixed } p} e^{\sum_{Q \subset V} \mathcal{L}_{Q}^{u}\left(\underline{\sigma}_{Q}\right)} Z(\Lambda /(V+R))}{\sum_{\text {at fixed } p} e^{\sum_{Q \subset V}-\mathcal{L}_{Q}^{s}\left(\underline{\sigma}_{Q}\right)} Z(\Lambda /(V+R))} e^{O(|\partial V|)}
$$

so that by the $(5.6),(5.8)$ and since the symmetry relations above imply $\sum_{Q \subset V}\left(\mathcal{L}_{Q}^{u}\left(\underline{\sigma}_{Q}\right)\right.$ $\left.+\mathcal{L}_{Q}^{s}\left(\underline{\sigma}_{Q}\right)\right)=V \eta_{+} p$, up to corrections of size $O\left(|\partial V| \kappa^{-1}\right)$ we find, (note the repetition of the comparison argument given in $[\mathrm{GC}])$ :

$$
\frac{\pi_{V}(p)}{\pi_{V}(-p)}=e^{\eta_{+} V p} e^{O(|\partial V|)}
$$

yielding a local fluctuation law.

Acknowledgments: I have profited of stimulating discussions with F. Perroni, who also helped with numerical tests of the above ideas, with F. Bonetto and D. Ruelle. This work is part of the research program of the European Network on: "Stability and Universality in Classical Mechanics", \# ERBCHRXCT940460; partially supported also by Rutgers University and CNR-GNFM. 


\section{References}

[An] Andrej, L.: The rate of entropy change in non-Hamiltonian systems, Physics Letters, 111A, 45-46, 1982.

[ES] Evans, Searles, : Equilibrium microstates which generate second law violating steady states, Physical Review E, 50E, 1645-1648, 1994.

[Ga1] Gallavotti, G.: Chaotic dynamics, fluctuations, nonequilibrium ensembles, Chaos, 8, 384-392, 1998.

[Ga2] Gallavotti, G.: New methods in nonequilibrium gases and fluids, Proceedings of the conference Let's face chaos through nonlinear dynamics, U. of Maribor, 24 June- 5 July 1996, ed. M. Robnik, in print in Open Systems and Information Dynamics, Vol. 5, 1998 (also in mp_arc@ math. utexas.edu \#96-533 and chao-dyn \#9610018).

[Ga3] Gallavotti, G.: Reversible Anosov maps and large deviations, Mathematical Physics Electronic Journal, MPEJ, (http:// mpej.unige.ch), 1, 12 pp., 1995.

[GC1] Gallavotti, G., Cohen, E.G.D: Dynamical ensembles in nonequilibrium statistical mechanics, Physical Review Letters, 74, 2694-2697, 1995. And Dynamical ensembles in stationary states, Journal of Statistical Physics, 80, 931-970, 1995.

[Ku] Kurchan, J.: Fluctuation theorem for stochastic dynamics, Journal of Physics A, 31, 3719-3729, 1998.

[LS] Lebowitz, J.L., Spohn, H.: The Gallavotti-Cohen Fluctuation Theorem for Stochastic Dynamics, preprint, Rutgers University, June 1998.

[MR] Morriss, G.P., Rondoni, L.: Applications of periodic orbit theory to $N$-particle systems, Journal of Statistical Physics, 86, 991, 1997.

[PS] Pesin, Y.B., Sinai, Y.G.: Space-time chaos in chains of weakly inteacting hyperbolic mappimgs, Advances in Soviet Mathematics, 3, 165-198, 1991.

[RT] Rom-Kedar, V., Turaev, D.: Big islands in dispersing billiard-like potentials, preprint, Weizmann Institute, April 2, 1998.

[Ru1] Ruelle, D.: Sensitive dependence on initial conditions and turbulent behavior of dynamical systems, Annals of the New York Academy of Sciences, 356, 408-416, 1978. [Ru2] Ruelle, D.: Positivity of entropy production in nonequilibrium statistical mechanics, Journal of Statistical Physics, 85, 1-25, 1996. And: Entropy production in nonequilibrium statistical mechanics, Comm. in Mathematical Physics, 189, 365-371, 1997.

[Si] Sinai, Y.: Lectures in ergodic theory, Lecture notes in Mathematics, Princeton U. Press, Princeton, 1977. 